\title{
EXPEDITIOUS CALCINATION OF INORGANIC MEMBRANES BY AN INSTANT TEMPERATURE INCREMENT
}

\author{
Emiel J. Kappert, Arian Nijmeijer, Nieck E. Benes* \\ Inorganic Membranes, Department of Science and Technology and MESA+ Institute for \\ Nanotechnology, University of Twente, P.O. Box 217, 7500 AE Enschede, The Netherlands \\ * Corresponding author: n.e.benes@utwente.nl, Tel: +31 534894288
}

\begin{abstract}
Rapid thermal treatments potentially allow for a significant reduction in production time of ceramic multilayered membranes, in turn aiding increased industrial application of these membranes and accelerating research on their development. Two methods are proposed for the rapid thermal treatment of thin supported inorganic membrane films. Both methods involve an instant increment in temperature imposed on the membrane. In the first method, the instant temperature step is enforced by placing the membrane in a preheated environment; in the second method, the membrane is placed directly onto a hot plate. The proposed methods can be used for a diverse range of materials. Mesoporous $\gamma$-alumina and microporous silica have been selected as model membrane materials. Both rapid heating methods require $\sim 20$ minutes to yield mesoporous $\gamma$-alumina membranes that are comparable to membranes made via conventional calcination ( $~ 1$ day). Selective silica membranes have been obtained after one hour exposure to an environment of $400{ }^{\circ} \mathrm{C}$ or $600{ }^{\circ} \mathrm{C}$, and after one hour contact with a hot plate of $550{ }^{\circ} \mathrm{C}$ (compared to up to 2 days for conventional calcination). The results indicate that, although prevention of contaminations needs continuous attention, both methods proposed for rapid heat treatment can reduce cost and time in ceramic membrane production.
\end{abstract}

Keywords: Rapid thermal processing, calcination, gas separation, sol-gel, $\gamma$-alumina, silica 


\section{Introduction}

Sol-gel deposition is a versatile method for the production of thin, inorganic membrane layers with a broad range of properties and competitive permeances and selectivities [1-7]. Sol-gel derived layers are coated on a porous support that provides mechanical strength. Multiple membrane layers are coated onto support sequentially, rendering the production process of the membranes laborious and expensive [8]. Especially the thermal treatment step is time consuming and energy-intensive. Typically, each layer is calcined at temperatures around $600{ }^{\circ} \mathrm{C}$ for 3 hours, with heating and cooling rates in the order of $1^{\circ} \mathrm{C}$ per minute $[4-7,9]$. For a full heating and cooling cycle from and to room temperature, this results in a total residence time in the furnace of 22 hours. For a membrane comprising multiple layers, processing time may continue for over a week. By minimizing the time required for thermal treatment, production costs of ceramic membranes can be decreased, in turn boosting opportunities for application and research for rapid material screening.

Recently, rapid thermal processing (RTP) was applied to inorganic membranes in the prevention of grain boundary defect formation in the zeolite silicate-1 [10] and for the significant reduction in calcination time of $\gamma$-alumina membranes [11]. In both cases, the application of RTP leads to an improvement in the material microstructure. While not yet commonly applied in membrane production, RTP is common in especially the semiconductor industry. Using an infrared lamp array, heating rates over $100{ }^{\circ} \mathrm{C}$ per second are applied in combination with dwell times shorter than ten minutes [12]. Apart from decreasing process time, there are several other reasons to choose for RTP over conventional processing, including the prevention of dopant diffusion [13], the improvement of crystallization behavior [14, 15], and the ability to rapidly fabricate multilayered films of micrometer thickness [16]. The high heating rate and controlled atmosphere give RTP an advantage over conventional furnace processing, especially for nanoscale systems that are sensitive to particles (e.g. dust, aerosols). 
Although thin membrane layers are known to be prone to defects, they may be less susceptible to contamination than e.g. integrated circuits, where one particle could damage the whole system. As RTP techniques have been developed for single wafers they may even be unsuited and too expensive for the production of membranes on industrial scales, where surface areas in the order of hundreds of square meters need to be treated thermally. In addition, RTP techniques seem inapt for thermal treatment of desired non-flat geometries like tubes and hollow fibers that are required for industrial up-scaling.

As an alternative to classic RTP methods, we demonstrate two generic rapid thermal treatment methods for the calcination of inorganic membranes. In both methods, an instant change in temperature is imposed onto the membrane. In the first method, hereafter referred to as Hot Environment (HE), the membrane is suddenly exposed to a preheated environment. In the second method, referred to as Hot Plate (HP), the membrane is placed directly onto a hot surface. Two model membranes were selected to explore the heating methods: supported thin film mesoporous $\gamma$-alumina, synthesized from a boehmite precursor containing an organic binder, and supported thin film microporous silica, synthesized from a polymeric sol in ethanol.

Silica membranes have arbitrarily been defined as ‘high-quality’ if selectivity for helium/methane is at least 100. From experience, it is known that such selectivities are only found on homogeneous $\gamma$-alumina intermediate layers with defect-free silica layers. Hence, by demonstrating the successful synthesis of high-quality, rapid-calcined $\gamma$-alumina and silica layers it is proven that rapid thermal treatments can be applied for the calcination of membrane layers without compromising their quality.

\section{Experimental}

\subsection{Materials}

AKP-30 $\alpha$-alumina powder (Sumitomo) was used for supports; aluminium tri-secbutylate (Merck) and poly(vinyl alcohol) (86.7-88.7 mol-\% hydrolysis, $M_{w} \sim 67,000$, 
Sigma-Aldrich) were used for boehmite sol synthesis and preparation of the coating solution; and tetraethyl orthosilicate (Merck) was used for silica sol synthesis. $\alpha$ alumina supports, made via a process identical to that of the homemade supports (described below), were purchased from Pervatech (Netherlands).

\subsection{Support and sol-synthesis}

Synthesis of flat $\alpha$-alumina supports, and synthesis of and coating with boehmite colloidal sol and silica sol was all carried out following the procedures described elsewhere [5]. Coating was carried out in a controlled environment (corresponding to clean room class 6).

\subsection{Calcination procedure}

Supported $\gamma$-alumina membranes were dried for three hours in a climate chamber (Heraeus Vötsch) at $40{ }^{\circ} \mathrm{C}$ and $60 \%$ relative humidity prior to thermal treatment. Thermal treatments were categorized into three groups: conventional calcination, hot environment calcination, and hot plate calcination. Conventional calcination was carried out in a furnace (Carbolite CWF with Eurotherm 3504 temperature controller) for three hours at the desired temperature with heating and cooling rates of $1{ }^{\circ} \mathrm{C}(\gamma$-alumina) or $0.5^{\circ} \mathrm{C}$ (silica), resulting in total calcination times of 22 and 41 hours, respectively. Hot environment calcination was carried out in the same furnace that was preheated to $400{ }^{\circ} \mathrm{C}$ or $600{ }^{\circ} \mathrm{C}$. Membranes were loaded onto a wafer and placed inside the furnace for the desired time. After removal, the wafer was placed on a thick alumina plate at room temperature to cool down. Hot plating was performed by placing membranes for desired times on a hot plate (Schott, SLK 1) at maximum power (corresponding to a surface temperature of approximately $550{ }^{\circ} \mathrm{C}$ ). During the hot plate experiment, the 
membrane was covered by a quartz glass plate to prevent contamination from the environment (Figure 1).

\section{Support ring}

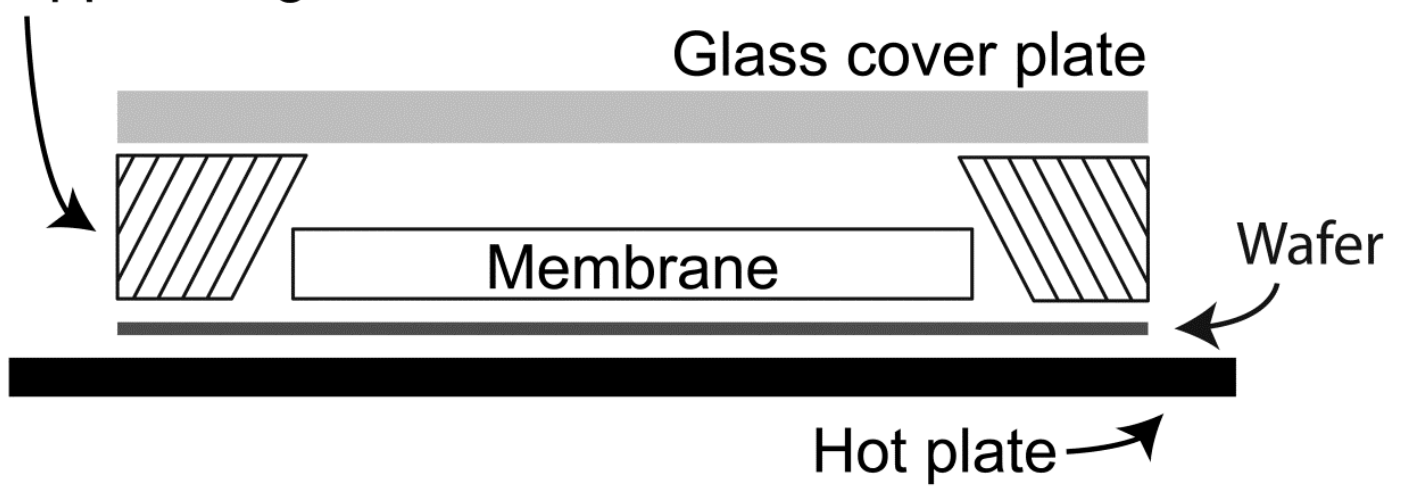

Figure 1: Covering of a membrane during hot plate experiments. The membrane is placed on a wafer and shielded by a quartz glass plate supported by an alumina ring.

\subsection{Nomenclature}

Conventionally calcined (CC) samples are indicated by material-temperature-CC; hot environment (HE) samples are named material-temperature-time; and hot plating (HP) samples are named material-temperature-time-HP. Materials are indicated by $\gamma(\gamma$ alumina) and Si (silica), temperature and time are in ${ }^{\circ} \mathrm{C}$ and minutes, respectively.

\subsection{Characterization}

The pore size of $\gamma$-alumina membranes was determined using permporometry. Measurements were carried out on an in-house designed apparatus using cyclohexane as the pore-filling liquid and an oxygen sensor to determine oxygen flow, following the procedure described by Cao et al. [17].

Silica membranes were characterized by single-gas permeation in dead-end mode without backpressure. The standard measurement carried out for every membrane 
consisted of measuring the permeances of a selection of $\mathrm{He}, \mathrm{H}_{2}, \mathrm{~N}_{2}, \mathrm{CO}_{2}, \mathrm{CH}_{4}$, and $\mathrm{SF}_{6}$ at $200{ }^{\circ} \mathrm{C}$ and a pressure difference of 2 bar. Single-gas permeance was determined by:

$$
F_{i}=\frac{N_{i}}{\Delta P}
$$

where $N$ is the molar flux through the membrane. The permselectivity $F_{\square}$ between a gas $i$ and a gas $j$ was determined by taking the ratio of the permeation of the two single gases.

To follow the debinding of organics from the $\gamma$-alumina precursors, video recordings of the membrane color in time were made with a Canon PowerShot A530 at 640 x 480 resolution and a frame rate of $10 \mathrm{~s}^{-1}$. The movie was cropped to a $15 \mathrm{x} 15$ pixel spot on the membrane. Using Matlab (version 2010a, Mathworks), RGB color data was extracted from the video and converted to $L^{*} a^{*} b^{*}$ color space (applying the makecform('srgb2lab') and applycform commands), after which the $L^{*} a^{*} b^{*}$ colors were averaged over the selected area. The color was compared to white ( $L^{*}=100, a^{*}=$ $0, b^{*}=0$ ) by taking the Euler distance between white and the measured color:

$$
\Delta E=\sqrt{\left(\Delta L^{*}\right)^{2}+\left(\Delta a^{*}\right)^{2}+\left(\Delta b^{*}\right)^{2}}
$$




\section{Results and discussion}

\section{1. $\gamma$-alumina membranes}

The obtained oxygen permeance through the different membranes was plotted as a function of the relative cyclohexane pressure in the gas in Figure 2A. For a non-cured $(\gamma-0)$ layer, no permeance was detected for the whole range of cyclohexane pressures, indicating a non-porous or microporous layer. For membranes rapidly calcined at increasing times, the cumulative oxygen permeance as well as the onset cyclohexane pressure at which permeance starts were found to increase as well.
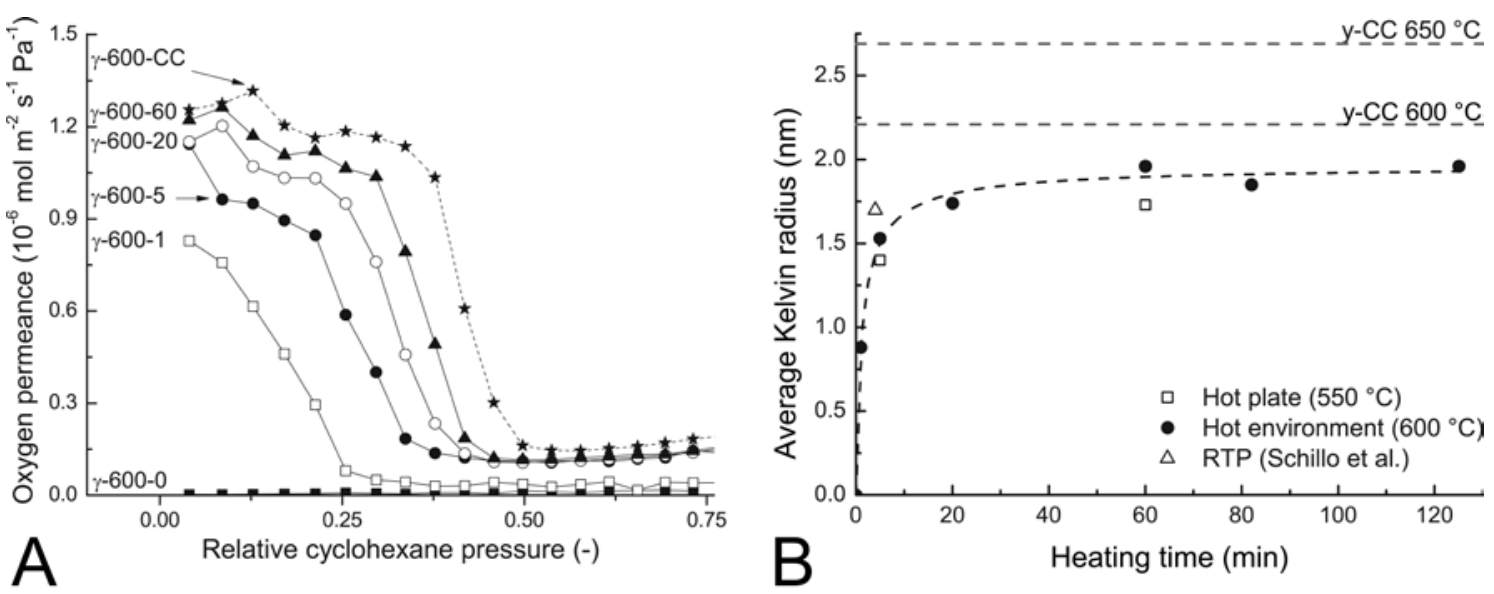

Figure 2: A. Oxygen permeance through rapidly calcined $\gamma$-alumina membrane layers as a function of relative cyclohexane pressure. B. Calculated average Kelvin radii for $\gamma$-alumina membranes after rapid treatment. The triangle indicates a $\gamma$-alumina layer after 3 minutes of RTP treatment as reported by Schillo et al. [11]

From the relative cyclohexane pressure, the corresponding Kelvin radius was calculated [17]. Figure 2B shows the progress of the average Kelvin radius of rapid-calcined $\gamma$ alumina membranes as a function of their thermal treatment time. The radius steeply increases in the first 5 minutes, after which the pore size slowly increases further to reach an asymptotic value of $2.0 \mathrm{~nm}$. The asymptotic Kelvin radius of $2.0 \mathrm{~nm}$ compares well to literature [18] and is only slightly lower than the average pore radius of 
conventionally calcined membranes (2.3 nm for $\gamma$-600-CC). Analysis of layers of boehmite/PVA on wafers by ATR-FTIR (see supplementary data) shows that no PVA remains in the structure after calcination. The results of this study also agree well to the results that were obtained by Schillo et al. using rapid thermal processing by a tungsten-halogen lamp array [11].

The steep pore size increase in the first five minutes of calcination is attributed to the debinding of poly(vinyl alcohol) from the membrane layer, which takes place in two steps. First, the alcohol groups are removed from the polymer backbone by chainstripping [19], resulting in a conjugated polyene with a brown-orange color. Secondly, at higher temperatures, the polyene will be decomposed into volatiles via a chainscission mechanism. In oxygen, this decomposition will not leave any residues behind if the temperature becomes higher than 700K [20]. Hence, to verify whether the fast increase in Kelvin radius in the first minutes indeed corresponds to the debinding of the poly(vinyl alcohol) binder from the green boehmite, the change in membrane color was recorded by performing the calcination step was performed on a hot plate and recording it on video. The derived $L^{*} a^{*} b^{*}$ color is plotted in Figure 3. 


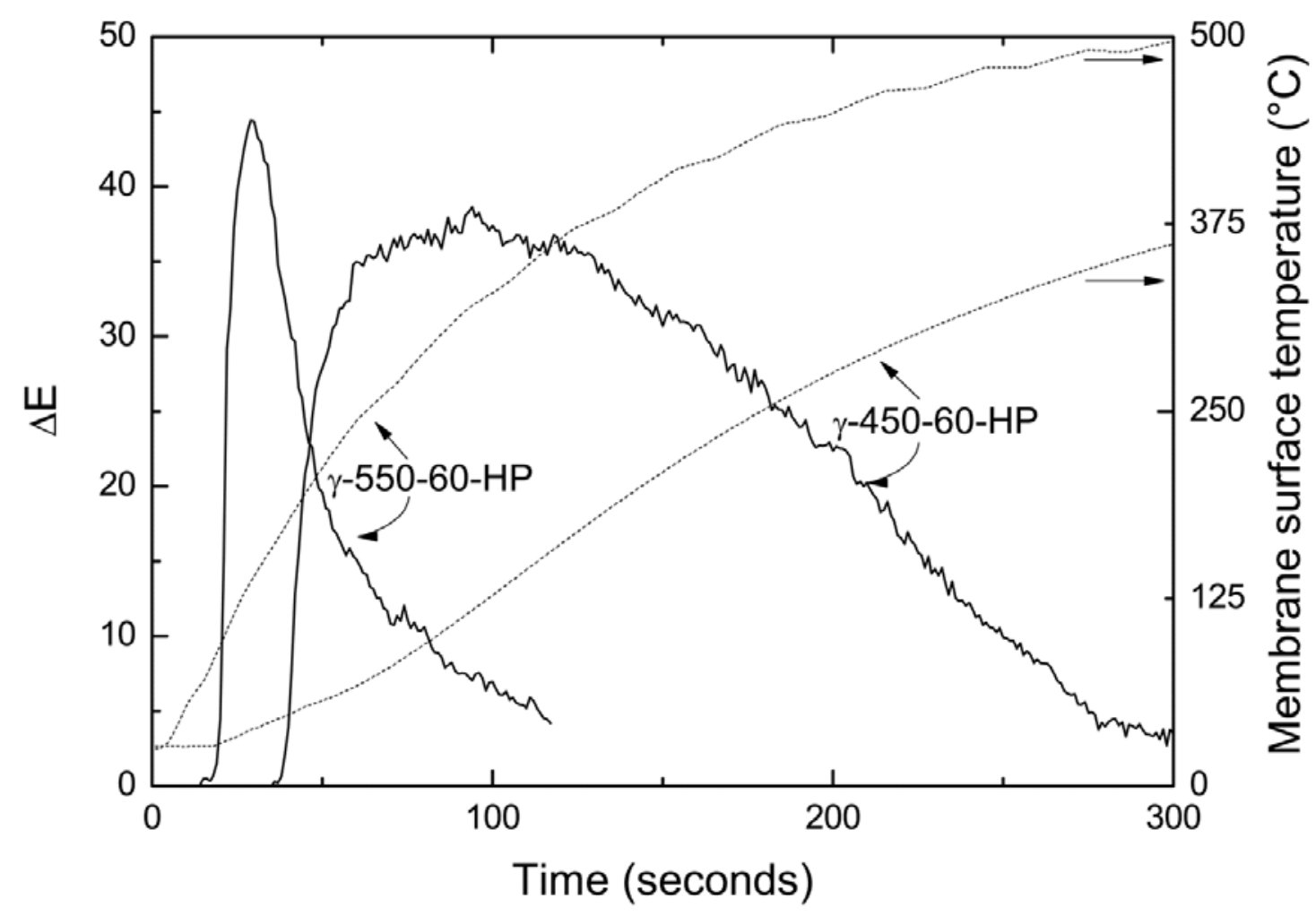

Figure 3: Membrane surface color (solid line) and temperature (dotted line) as a function of the time on the hot plate. The color is given as the deviation from white as determined by equation 2.

The progress of color and temperature in time is visualized for two membranes: $\gamma-550$ 60-HP and $\gamma$-450-60-HP, where a 2 mm copper plate was inserted as a thermal barrier between the hot plate and the membrane in the latter case. In both cases, a temperaturecolor correlation is evident, since the darkest color is observed as the membrane surface reaches a temperature of $150{ }^{\circ} \mathrm{C}$ and the membrane returns to its initial color at roughly $400{ }^{\circ} \mathrm{C}$.

\subsection{Rapidly calcined silica layers}

The permeance of multiple gases through Si-400-60 and Si-600-60 membranes at $200{ }^{\circ} \mathrm{C}$ is given in Figure 4 and Figure 5, respectively. Regardless of the temperature, 
the membranes treated for 60 minutes show comparable helium permeances, averaging $3.13 \pm 0.75 \cdot 10^{-7} \mathrm{~mol} \mathrm{~m}^{-2} \mathrm{~s}^{-1} \mathrm{~Pa}^{-1}$. This value compares well to literature, indicating that applying an instant temperature step did not result in significant network densification. The high permselectivity of the silica layers $\left(\mathrm{He} / \mathrm{CH}_{4}>100\right)$ indicates a defect-free layer and thus demonstrates the viability of the hot environment technique. On the other hand, as can be seen in Figures 4 and 5, approximately 50\% of the membranes only show moderate selectivities ( $\mathrm{He} / \mathrm{CH}_{4}<100$, henceforth called defect), whereas for conventionally processed membranes, a defect rate of approximately $15 \%$ is stated [7]. This increased defect rate for the HE method can be explained by the increased exposure to particles present in the environment (e.g. dust, aerosols) that damage the thin membrane layer. Whereas this contamination is easily prevented in the conventional process by shielding the membranes until placement in the furnace, the shielding requires more attention in the case of a preheated furnace. As we focused on delivering a proof-of-concept, no effort was taken to optimize the shielding for the hot environment concept.

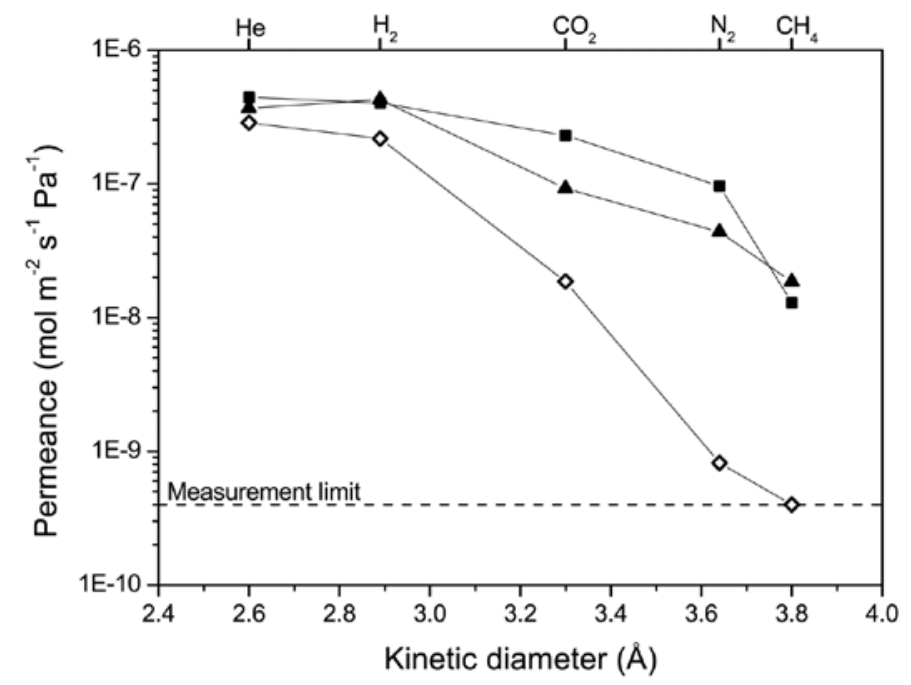

Figure 4: Permeance through silica layers rapidly calcined in a hot environment at $400{ }^{\circ} \mathrm{C}$ for 60 minutes (Si-400-60). The three lines indicate distinct membranes that were made through identical procedures. 


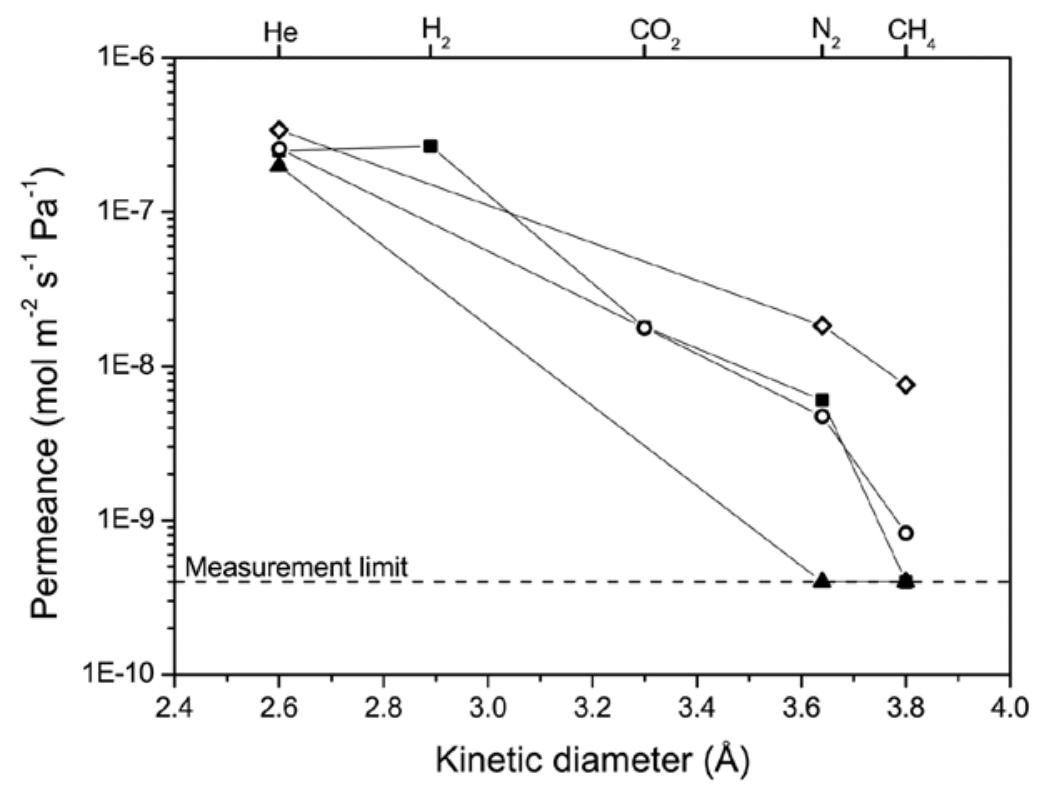

Figure 5: Permeance through silica layers rapidly calcined in a hot environment at $600{ }^{\circ} \mathrm{C}$ for 60 minutes (Si-600-60). The four lines indicate distinct membranes that were made through identical procedures.

Figure 6 shows the permeances of a batch of membranes calcined for only 10 minutes at $600{ }^{\circ} \mathrm{C}$ (Si-600-10). Although the helium permeance of these membranes equals that of the membranes calcined for 60 minutes, the selectivity of helium over nitrogen and methane is low as compared to conventional membranes [6] and the membranes calcined for 60 minutes. Apparently, the desired membrane microstructure has not yet been fully developed after 10 minutes. 


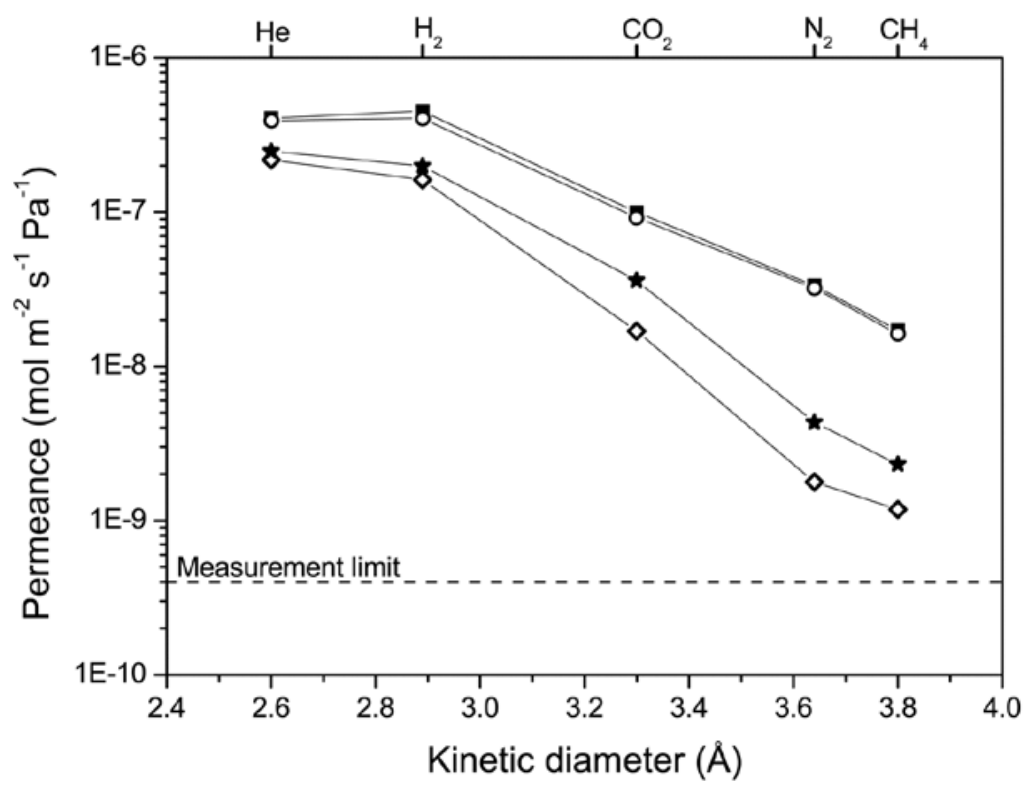

Figure 6: Permeance through silica layers rapidly calcined in a hot environment at $600{ }^{\circ} \mathrm{C}$ for 10 minutes (Si-600-10). The four lines indicate distinct membranes that were made through identical procedures.

Figure 7 shows the permeance data of a membrane obtained after 1 hour calcination on a hot plate temperature of $550{ }^{\circ} \mathrm{C}$. The helium permeance is comparable to that of the other membranes. The measured selectivity of $\mathrm{He} / \mathrm{CO}_{2}$ is 43 , and the selectivities of $\mathrm{He} / \mathrm{N}_{2}$ and $\mathrm{He} / \mathrm{CH}_{4}$ are well above 500 . These values show that rapid calcination using the hot plate can result in high-performance membranes. It has to be noted that continuous attention needs to be paid to the prevention of contamination; any calcination performed without the shielding device (Figure 1) resulted in membranes showing only Knudsen selectivity (even for sulfur hexafluoride, $d_{k}=5.5 \AA$ ), which indicate pores with sizes larger than nanometers, or defects. 


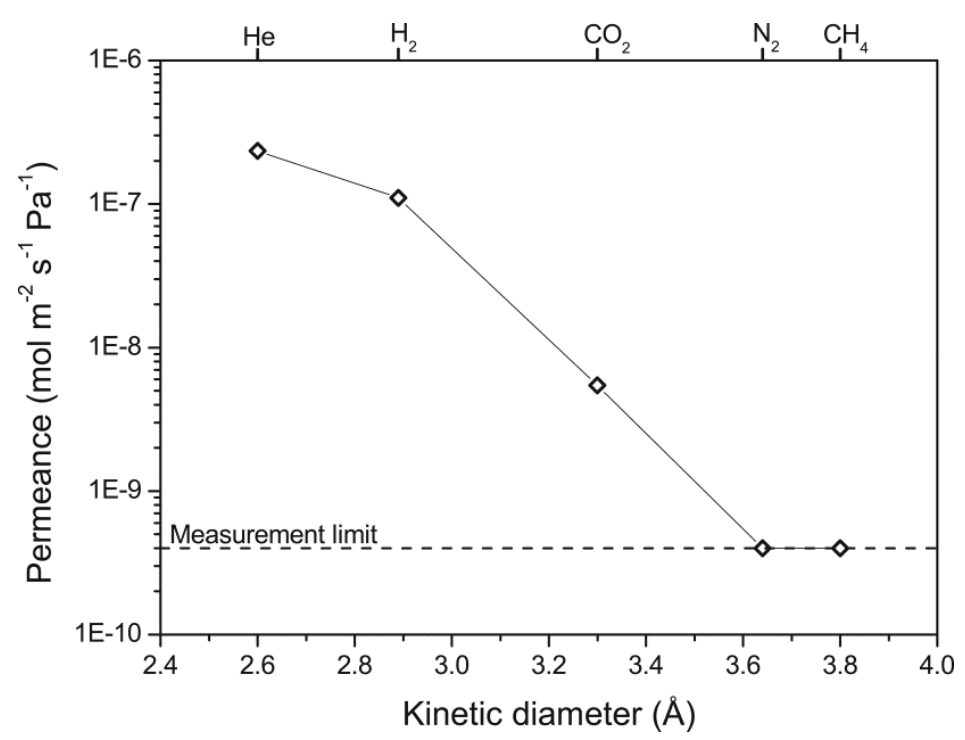

Figure 7: Gas permeance data obtained on the hot plate at $550{ }^{\circ} \mathrm{C}$ for 1 hour (Si550-60-HP) using the cover mechanism.

\section{Conclusions}

Rapid thermal treatments are employed successfully for the calcination of $\gamma$-alumina and silica membranes. The membranes obtained by the proposed methods are comparable to conventionally calcined membranes; however, the calcination time is reduced from 22 hours to 30 minutes for $\gamma$-alumina, and from 41 hours to at most 1 hour for silica. This is a reduction to less than $3 \%$. For both methods, a decrease in repeatability of the experiments was found, presumably caused by defects in the membrane layers that originate from an increased exposure time to the environment at the insertion and removal of the membranes from the furnace. Likely, this defect rate can be decreased in an optimized configuration, where the shielding of the membrane from the environment is further improved.

$\gamma$-alumina and silica are regarded representative for meso- and microporous membrane layers, respectively. Since both types of materials where successfully calcined rapidly, 
the developed rapid thermal treatment methods can probably be extended to a wide class of inorganic materials, like titania and hybrid organic-inorganic membranes. Employing rapid thermal treatments encourages the rapid screening of materials for research purposes. Above all, on industrial scale, both methods offer an advantage over RTP, as they can be scaled to larger areas and applied to non-planar geometries, potentially decreasing costs and production time. The results confirm the viability of rapid thermal treatments and give reason to continue the research into a wider range of materials and further optimized configuration for hot plate experiments.

\section{References}

[1] A.F.M. Leenaars and A.J. Burggraaf, J. Colloid Interface Sci., 105 (1985) 27.

[2] A. Julbe, C. Guizard, A. Larbot, L. Cot and A. Giroir-Fendler, J. Membr. Sci., 77 (1993) 137.

[3] T. Van Gestel, C. Vandecasteele, A. Buekenhoudt, C. Dotremont, J. Luyten, R. Leysen, B. Van der Bruggen and G. Maes, J. Membr. Sci., 207 (2002) 73.

[4] R.J.R. Uhlhorn, M.H.B.J. Huis In 't Veld, K. Keizer and A.J. Burggraaf, J. Mater. Sci. Lett., 8 (1989) 1135.

[5] N.E. Benes, A. Nijmeijer and H. Verweij, in N.K. Kanellopoulos (Editor), Recent advances in gas separation by microporous ceramic membranes, Elsevier, Amsterdam, 2000.

[6] Y.S. Lin, I. Kumakiri, B.N. Nair and H. Alsyouri, Sep. \& Purif. Rev., 31 (2002) 229.

[7] R.M. de Vos and H. Verweij, Science, 279 (1998) 1710.

[8] S. Gopalakrishnan, B.N. Nair and S.-I. Nakao, International Conference on Nanoscience and Nanotechnology 3-7 July 20062006.

[9] D. Uhlmann, S. Liu, B.P. Ladewig and J.C. Diniz da Costa, J. Membr. Sci., 326 (2009) 316.

[10] J. Choi, H.-K. Jeong, M.A. Snyder, J.A. Stoeger, R.I. Masel and M. Tsapatsis, Science, 325 (2009) 590.

[11] M.C. Schillo, I.S. Park, W.V. Chiu and H. Verweij, J. Membr. Sci., 362 (2010) 127.

[12] P.J. Timans, in R. Doering and Y. Nishi (Editors), Handbook of semiconductor manufacturing technology, CRC Press, Boca Raton, 2008.

[13] R.B. Fair, Proceedings of the IEEE, 78 (1990) 1687.

[14] Y. Motemani, M.J. Tan, T.J. White and A. Banas, Surf. Coat. Technol., 205 (2011) 3147.

[15] K. Siemer, J. Klaer, I. Luck, J. Bruns, R. Klenk and D. Bräunig, Sol. Energy Mater. Sol. Cells, 67 (2001) 159.

[16] H.-K. Jeong, R. Chandrasekharan, K.-L. Chu, M.A. Shannon and R.I. Masel, Ind. Eng. Chem. Res., 44 (2005) 8933. 
[17] G.Z. Cao, J. Meijernik, H.W. Brinkman and A.J. Burggraaf, J. Membr. Sci., 83 (1993) 221.

[18] F.P. Cuperus, D. Bargeman and C.A. Smolders, J. Membr. Sci., 71 (1992) 57.

[19] J.W. Gilman, D.L. VanderHart and T. Kashiwagi, ACS Symposium Series 599, Washington, DC, 1994.

[20] C.L. Beyler and M.M. Hirschler, SFPE Handbook of Fire Protection Engineering, 3rd ed., 2002, p. 110. 


\section{Figure captions}




\section{Figures}

Figure 7 